\title{
Steady Magnetohydrodynamic flow of Third- Grade Non-Newtonian Nanofluid in Porous Coaxial Cylinders
}

\author{
Zubaidah Sadikin* and Seripah Awang Kechil
}

\author{
Faculty of Computer and Mathematical Sciences, UiTM Shah Alam, Malaysia
}

\begin{abstract}
In this paper, the transport processes in magnetohydrodynamics third-grade non-Newtonian nanofluids in porous coaxial cylinders is studied. The effects of the physical parameters particularly the thermophoresis parameters, Brownian motion, third grade parameter and magnetic parameter on the velocity, temperature, nanoparticles concentration, and drugs concentration are analysed. The transformed system of nonlinear ordinary differential equations is solved numerically using the fourthorder Runge-Kutta method. The viscosity of the nanofluid is considered as a function of temperature in form of Vogel's model. Numerical solutions are obtained for the velocity, temperature, nanoparticles concentration and drugs concentration. It is found that increasing the thermophoresis parameter leads to the increase in temperature while the velocity and nanoparticles and drug distributions decrease. The increment of the thermophoresis parameter generates larger mass flux due to temperature gradient, which in turn reduce the nanoparticle concentration and drug concentration near the wall.
\end{abstract}

Keywords: Non-Newtonian nanofluid, MHD, Thermophoresis, Brownian motion

\section{INTRODUCTION}

Current development of nanotechnology significantly advances the fields of information technology, environmental science, medicine, security, transportation, energy and food safety, among many others. Engineered nanoparticles are used as nanocarriers to transport specific substance such as drug, heat or light to targeted areas. The transport processes of momentum, nanoparticles, heat and species concentration in a system can be actively controlled by physical stimuli such as temperature, $\mathrm{pH}$ and magnetism or passively controlled through permeability and retention. Empirical models of these transport processes assume a single-zeroorder procedure while deterministic models consider all the mass transport phenomena including the flow and transfer rates, the species diffusion and chemical reaction processes (Siepmann, 2001). Hossain et. al., (2012) developed a comprehensive $3 \mathrm{D}$ advection-diffusion-reaction model to provide the generality of the physiological and transport phenomena in the catheter-based lumen side delivery system. They found that off target nanoparticles contribute significantly to the overall therapeutic effects and thicker wall due to the existence of plaque reduces the drug concentration level in the neighbouring healthy regions.

The transport processes in nanofluids can be enriched by the consideration of the base fluid of differential type that take into account the nonlinear relation between the shear stress and rate of shear strain. Third grade fluid exhibits shear thickening and thinning behaviour. Fosdick et al. (1980) was the first to analyse the thermodynamical properties of third-grade fluid.

Applied magnetics force, chemical reaction and heat generation or absorption are significant mechanisms to alter the behaviour of nanofluid flows. Hayat et al. (2015) studied the MHD axisymmetric flow of third grade fluid by a stretching cylinder. Usman et. al., (2016) investigated the fluid flow and heat transfer of blood with 
nanoparticles through porous vessels in the presence of magnetic field. Hayat et. al., (2016) examined the chemical reactive flow of third grade fluid over an exponentially convected stretching sheet in the presence of magnetic field. Mekheimer et al. (2018) examined the peristaltic blood flow with gold nanoparticles as a third grade nanofluid in catheter in the application of cancer therapy. The unsteady pulsatile magnetohydrodynamic blood flows through porous arteries in concerning the influence of externally imposed periodic body acceleration and a periodic pressure gradient using a third-grade non-Newtonian fluids model was investigated numerically by Akbarzadeh et. al., (2018).

The aim of this paper is to study the magnetohydrodynamic of third-grade non-Newtonian nanofluid flow through porous coaxial cylinders, in the presence of nanoparticles acting as drug nanocarriers. The effects of magnetic parameter or also known as Hartmann number $\left(M^{2}\right), \quad$ third-grade $\quad$ parameter $(\Lambda)$, thermophoresis parameter $\left(N_{T}\right)$, Brownian motion parameter $\left(N_{B}\right)$ on the velocity, temperature, nanoparticle and drug concentration distributions are analysed. This study extends the works of Hatami et al. (2014) and Hossain et. al., (2012) to include the case of the mass transfer of the nanoparticles and drug concentration. The numerical solution of the boundary layer equations by fourth-order Runge-Kutta method with shooting technique are obtained and discussed for several values of the nanofluid parameters. The system of the higher-order ordinary differential equations is reduced to system of first-order ordinary differential equations. The endpoints of the boundaries are divided to subintervals with its step-size. The unknown values are approximated until the matched the conditions at the surface of the outer cylinder. Thus, the combined effects of momentum and concentration transport with metabolic decay are examined.

\section{MATHEMATICAL FORMULATION}

In this section, the mathematical formulation of the governing equations of mass, momentum, thermal energy, diffusion of nanoparticles concentrations and advection of drug concentrations are derived.

Consider a steady, incompressible and electrically conducting third grade non-Newtonian nanofluids of twodimensional boundary layer flow over a porous coaxial cylinder in the presence of an applied magnetic field.

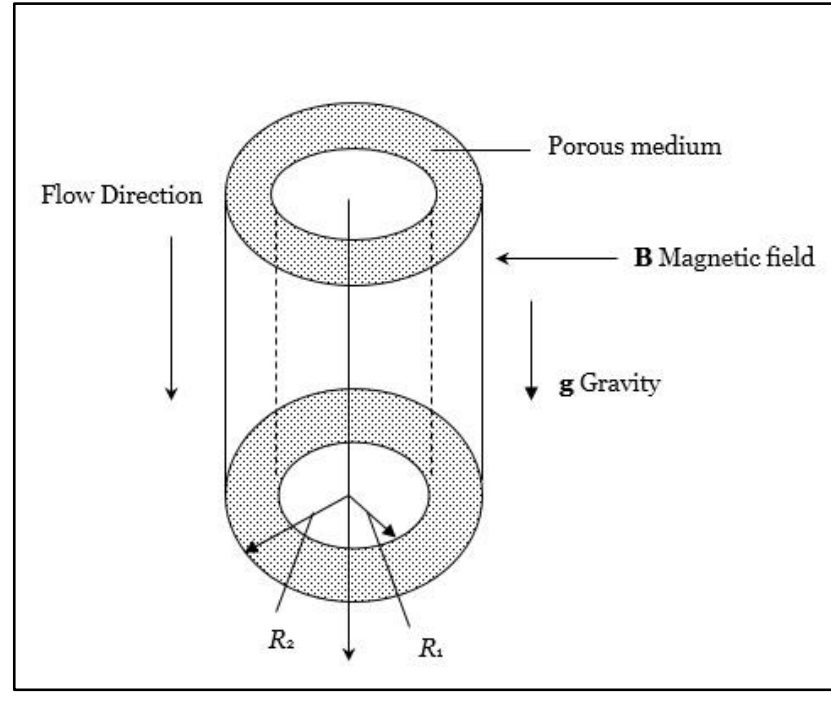

Figure 1. Physical model of problem

The physical model in Figure 1 shows the cylindrical coordinates where the radius of the inner cylinder is $R_{1}$ and the radius of the outer cylinder is $R_{2}$. For natural convection, the nanofluid's density of Buongiorno (2006) is

$\rho \cong \phi \rho_{p}+(1-\phi) \rho_{f}\left(1-\beta_{T}\left(T-T_{w}\right)\right)$

where $\phi$ is the nanoparticles volume fraction, $T$ is the temperature, $\rho_{f}$ is the base fluid's density at the reference temperature, $\rho_{p}$ is the density of nanoparticles, $\beta_{T}$ is the volumetric coefficient of expansion and $T_{w}$ is temperature at the surface of the inner cylinder.

The viscosity $\mu$ is assumed to be a function of temperature according to Vogel's viscosity model Ellahi et al. (2012)

$$
\mu(T)=\mu_{\mathrm{o}} \exp \left(\frac{A}{B+T}-T_{\mathrm{o}}\right)
$$

where $\mu_{\mathrm{o}}$ is the viscosity at the reference temperature $T_{\mathrm{o}}$, $A=a /\left(T_{m}-T_{w}\right)$, and

$$
B=\left(b+T_{w}\right) /\left(T_{m}-T_{w}\right) \text { where } a \text { and } \quad b \text { are }
$$

constants, $T_{m}$ is the fluid's temperature.

\section{A. Governing Equations}

The nanoparticles are assumed to be uniform in shape and size. The magnetic Reynolds number is assumed to be 
small so that the induced magnetic field is negligible in comparison to the applied magnetic field. We also assume that there is no applied electric field. The Hall effect, Joule heating and viscous dissipation are considered small and negligible. Under the assumption of the OberbeckBoussinesq approximation, assuming dilute nanoparticle concentration and with suitable reference pressure and boundary layer approximation, the steady MHD boundary layers in non-Newtonian nanofluid are governed by the conservation of momentum and thermal energy (Hatami et. al., 2014; Hossain et. al., 2012),

$$
\begin{aligned}
& \rho_{f}\left(\frac{\partial \mathbf{V}}{\partial t}+(\mathbf{V} \cdot \nabla) \mathbf{V}\right)=\operatorname{div} \tau-\frac{\mu \varphi}{K}\left(1+\lambda_{r} \frac{\partial}{\partial t}\right) \mathbf{V} \\
& +\left[\left(\rho_{p}-\rho_{w}\right)\left(\phi-\phi_{w}\right)+\left(1-\phi_{w}\right) \rho_{w} \beta\left(T-T_{w}\right)\right] \mathbf{g} \\
& -\sigma B_{\mathrm{o}}^{2} \mathbf{V} .
\end{aligned}
$$$$
(\rho c)_{f}\left(\frac{\partial T}{\partial t}+(\mathbf{V} \cdot \nabla) T\right)=k \nabla^{2} T
$$$$
+(\rho c)_{p}\left[D_{B} \nabla \phi \cdot \nabla T+\frac{D_{T}}{T} \nabla T \cdot \nabla T\right] .
$$

where $v(r)$ is the velocity vector field, $\mu$ is the viscosity, $\varphi$ is porosity of medium, $\tau$ is the stress in third-grade fluid, $k$ is the thermal conductivity of the nanofluids, $K$ is the permeability of porous medium, $\lambda_{r}$ is the retardation time, $\rho_{w}$ is the base fluid's density at the reference temperature, $\sigma$ is the electrical conductivity, $(\rho c)_{f}$ is the heat capacity of the fluid, $(\rho c)_{p}$ is the effective heat capacity of the nanoparticles, $\mathbf{g}$ is gravitational vector, $D_{B}$ is the Brownian diffusion coefficient, $D_{T}$ is the thermophoresis diffusion coefficient and $\phi_{w}$ is the wall concentration.

The stress $\tau$ in a third-grade fluid is

$$
\begin{aligned}
\tau= & -p_{1} I+\mu \mathbf{A}_{1}+\alpha_{1} \mathbf{A}_{2}+\alpha_{2} \mathbf{A}_{1}^{2}+\beta_{1} \mathbf{A}_{3} \\
& +\beta_{2}\left(\mathbf{A}_{1} \mathbf{A}_{2}+\mathbf{A}_{2} \mathbf{A}_{1}\right)+\beta_{3}\left(\operatorname{tr} \mathbf{A}_{1}^{2}\right) \mathbf{A}_{1}
\end{aligned}
$$

where the material modules $\alpha_{1}, \alpha_{2}, \beta_{1}, \beta_{2}$, and $\beta_{3}$ are considered to be functions of temperature. The term $-p_{1}$ in (5) shows the spherical stress due to the restraint of incompressibility. The kinematical tensors $\mathbf{A}_{1}, \mathbf{A}_{2}$ and $\mathbf{A}_{3}$ are defined by following equations,

$$
\begin{gathered}
\mathbf{A}_{1}=(\nabla \mathbf{V})+(\nabla \mathbf{V})^{t r} \\
\mathbf{A}_{n}=\frac{D \mathbf{A}_{(n-1)}}{D t}+\mathbf{A}_{(n-1)}(\nabla \mathbf{V})+(\nabla \mathbf{V})^{t r} \mathbf{A}_{(n-1)}
\end{gathered}
$$

Where $\mathbf{V}=[\mathrm{O}, \mathrm{O}, v(r)]$ denotes the velocity field, $t r$ denotes the transpose and $D / D t$ is the material time derivative defined by $D(.) / D t=\partial(.) / \partial t+[\operatorname{grad}().] \mathbf{V}$ ,grad is the gradient operator.

When the fluid is locally at rest, the specific Helmholtz free energy is at minimum (Fosdick et. al., 1980)

$$
\begin{gathered}
\mu \geq 0, \quad \alpha_{1} \geq 0, \quad\left|\alpha_{1}+\alpha_{2}\right| \leq \sqrt{24 \mu \beta_{3}}, \\
\beta_{1}=\beta_{2}=\mathrm{o}, \quad \beta_{3} \geq 0 .
\end{gathered}
$$

If the fluid is thermodynamically compatible, (5) is reduced to

$$
=-p_{1} I+\left(\mu+\beta_{3}\left(\operatorname{tr} \mathbf{A}_{1}^{2}\right)\right) \mathbf{A}_{1}+\alpha_{1} \mathbf{A}_{2}+\alpha_{2} \mathbf{A}_{1}^{2} .
$$

We also consider the mass transport of nanocarriers and drug are governed by the diffusive, advective and reactive processes. These transport processes are sensitive to temperature and concentration gradients. The metabolic decay of drug and nanocarriers are assumed to follow a first order reaction. Thus, the conservation of nanoparticles concentration with the effect of temperature is

$\left(\frac{\partial \phi}{\partial t}+(\mathbf{V} \cdot \nabla) \phi\right)=D_{B} \nabla^{2} \phi+\frac{D_{T}}{T} \nabla^{2} T-\sigma_{1}\left(\phi-\phi_{w}\right)$

where $D_{B}$ is the Brownian diffusion coefficient and $D_{T}$ is the thermophoresis diffusion coefficient. The equation of drug concentration is

$$
\begin{aligned}
\left(\frac{\partial C}{\partial t}+(\mathbf{V} \cdot \nabla) C\right)= & -\nabla \cdot D_{C} \nabla C-\sigma_{2}\left(C-C_{w}\right) \\
& +\left(\phi-\phi_{w}\right) f
\end{aligned}
$$

where $D_{C} \nabla C$ is the anisotropic diffusion of drug concentration, $C$. The terms $(\mathbf{V} \cdot \nabla) \phi$ and $(\mathbf{V} \cdot \nabla) C$ represent the advection process. The terms $-\sigma_{1}\left(\phi-\phi_{w}\right)$ and $-\sigma_{2}\left(C-C_{w}\right)$ are the reaction term for metabolic decay of nanoparticles and drug, respectively. The two transport equations (9) and (10) are coupled through the source term $\left(\phi-\phi_{w}\right) f$ for the constant drug release rate from nanoparticles (Hossain et al., 2012). 
The boundary conditions of the governing equations

$$
\text { at } \begin{aligned}
r & =R_{1} \\
v & =v_{\mathrm{o}}, \quad T=T_{w}, \quad \phi=\phi_{w}, \quad C=C_{w}
\end{aligned}
$$

and at $r=R_{2}$

$$
v=0, \quad T=0, \quad \phi=0, \quad C=0 .
$$

\section{B. Nondimensional Equations}

Following Hatami et. al., (2014) we introduce the nondimensional parameters $v_{0}, \mu_{\mathrm{o}}, R_{1}, T_{w}$ and $T_{m}$ to denote the reference velocity, reference viscosity, reference radius, ambient temperature, and fluid temperature, respectively. Therefore, the dimensionless variables are

$$
\begin{aligned}
& \bar{v}=v / v_{o}, \bar{T}=\left(T-T_{w}\right) /\left(T_{m}-T_{w}\right), \\
& \bar{\phi}=\left(\phi-\phi_{w}\right) /\left(\phi_{m}-\phi_{w}\right), \\
& \bar{C}=\left(C-C_{w}\right) /\left(C_{m}-C_{w}\right), \\
& \bar{r}=r / R_{1}, \bar{\mu}=\mu / \mu_{o} .
\end{aligned}
$$

The system (3), (4), (9\}) - (12) with assumptions (2\}) and (8\}) under the transformation (13) and dropping the bar,

$$
\begin{aligned}
& \frac{d \mu(r)}{d r} \frac{d v(r)}{d r}+\frac{\mu(r)}{r} \frac{d v(r)}{d r}+\mu(r) \frac{d^{2} v(r)}{d r^{2}} \\
& +3 \Lambda\left(\frac{d v(r)}{d r}\right)^{2} \frac{d^{2} v(r)}{d r^{2}}+\frac{\Lambda}{r}\left(\frac{d v(r)}{d r}\right)^{3} \\
& =P \mu(r) v(r)+M^{2} v(r)+c-B_{r} \phi(r)-G_{r} T(r), \\
& \alpha\left(\frac{d^{2} T(r)}{d r^{2}}+\frac{1}{r} \frac{d T(r)}{d r}\right) \\
& +\alpha_{1}\left[N_{B} \frac{d \phi(r)}{d r} \frac{d T(r)}{d r}+N_{T}\left(\frac{d T(r)}{d r}\right)^{2}\right]=\mathrm{O} \\
& N_{B}\left(\frac{d^{2} \phi(r)}{d r^{2}}+\frac{1}{r} \frac{d \phi(r)}{d r}\right) \\
& +N_{T}\left(\frac{d^{2} T(r)}{d r^{2}}+\frac{1}{r} \frac{d T(r)}{d r}\right)-D a_{1} \phi(r)=\mathrm{O} \\
& \frac{d^{2} C(r)}{d r^{2}}+\frac{1}{r} \frac{d C(r)}{d r}-D a_{2} C(r)+\phi(r) f=0
\end{aligned}
$$

with boundary conditions

$$
\begin{aligned}
& v(1)=1, \quad T(1)=1, \quad \phi(1)=1, \quad C(1)=1, \\
& \quad v(2)=0, \quad T(2)=0, \quad \phi(2)=0, \quad C(2)=0 .
\end{aligned}
$$

Following Ellahi et. al., (2011), Hatami et. al., (2014) and Kuznetsof et. al., (2010) the non-dimensional variables are the third grade parameter $\Lambda=\frac{2 \beta_{3} v_{o}^{2}}{\mu_{o} R_{1}^{2}}$, porosity $P=\frac{\varphi R_{1}^{2}}{K}$, pressure gradient $c=\frac{(\partial p / \partial z) R_{1}^{2}}{\mu_{o} v_{o}}$, thermal diffusivity $\alpha=\frac{k}{(\rho c)_{f}}$, ratio of effective heat capacity of nanoparticles and fluid $\alpha=\frac{(\rho c)_{p}}{(\rho c)_{f}}$, Brownian diffusion constant $B_{r}=\frac{\left(\rho_{p}-\rho_{w}\right) R_{1}^{2}\left(\phi_{m}-\phi_{w}\right) g}{\mu_{o} v_{o}}$,

Thermophoresis diffusion $G_{r}=\frac{\left(T_{m}-T_{w}\right) \rho_{w} R_{1}^{2}\left(1-\phi_{w}\right) g}{\mu_{o} v_{o}}$, Magnetic parameter $M^{2}=\frac{\sigma B_{o}^{2} R_{1}^{2}}{\mu_{o}}$, Brownian motion parameter $N_{B}=D_{B}\left(\phi_{m}-\phi_{w}\right)$, Thermophoresis parameter $N_{T}=\frac{D_{T}\left(T_{m}-T_{w}\right)}{T_{w}}$, Damkohler number of nanoparticles concentration $D a_{1}=\frac{\sigma_{1} R_{1}^{2}}{D_{B}}$, and Damkohler number of drug concentration $D a_{2}=\frac{\sigma_{1} R_{1}^{2}}{D_{C}}$

\section{RESULT AND DISCUSSIONS}

It is observed that the velocity, temperature, nanoparticles concentration and drug concentration decrease as $r$ increases as demonstrated in Figure 2. The velocity and temperature profiles for various values of the thermophoresis parameter are illustrated in Figure $3 \mathrm{a}$ and $3 \mathrm{~b}$, respectively. Little effects of $N_{T}$ is observed on the velocity, $v(r)$ where $v(r)$ decreases as $N_{T}$ increases. An increment in $N_{T}$ enhances the temperature. Thermophoresis is a mechanism in which the heated particles move away from hot surface to the cold region, resulting in rising temperature of the fluid farther from the wall. Figure $3 c-3 d$ show that by increasing the values of $N_{T}$, the nanoparticles concentration, $\phi(r)$ and drug 
concentration, $C(r)$ decrease.

The velocity and temperature profiles for various value of the Brownian motion parameter are illustrated in Figure $4 \mathrm{a}$ and Figure $4 \mathrm{~b}$. The velocity, $v(r)$ and temperature $T(r)$ increase as $N_{B}$ increases. The results also show that by increasing the values of $N_{B}$, the nanoparticles concentration, $\phi(r)$ and drug concentration, $C(r)$ increase as illustrated in Figure 4c-4d. Higher Brownian motion $N_{B}$ will result in the transfer of heat by the random motion of nanoparticles. Increasing Brownian motion parameter $N_{B}$ and thermophoresis parameter $N_{T}$, thicken the thermal boundary layers, however, increasing $N_{T}$ or decreasing $N_{B}$ decreases the velocity boundary layer thickness.

Figure $5 \mathrm{a}-5 \mathrm{~b}$ depict the effect of third grade parameter, $\Lambda$ and magnetic parameter, $M^{2}$ or Hartmann numbers on the velocity. Larger $\Lambda$ shows reduction in the velocity boundary layer thickness. Hartmann number measures the relative strength of Lorentz force to viscous hydrodynamic force and increasing the Hartmann number will result in viscous drag that will decrease the velocity.

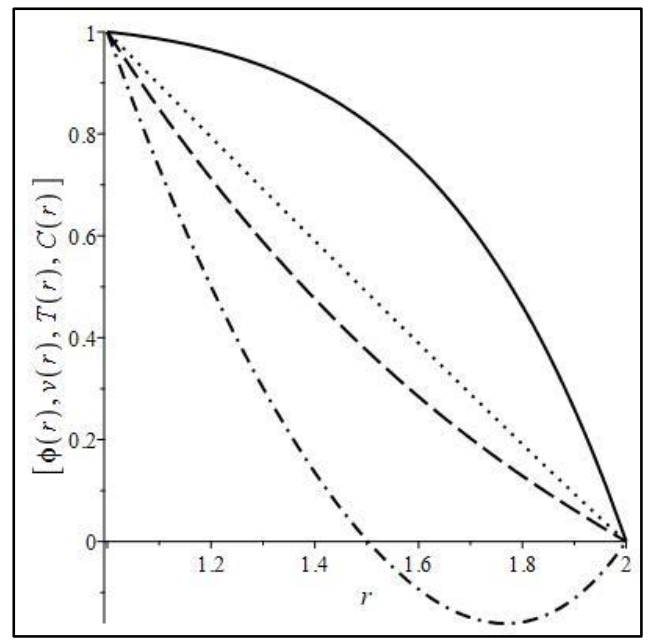

Figure 2. The profile for velocity, temperature, nanoparticles concentration and drug concentration.

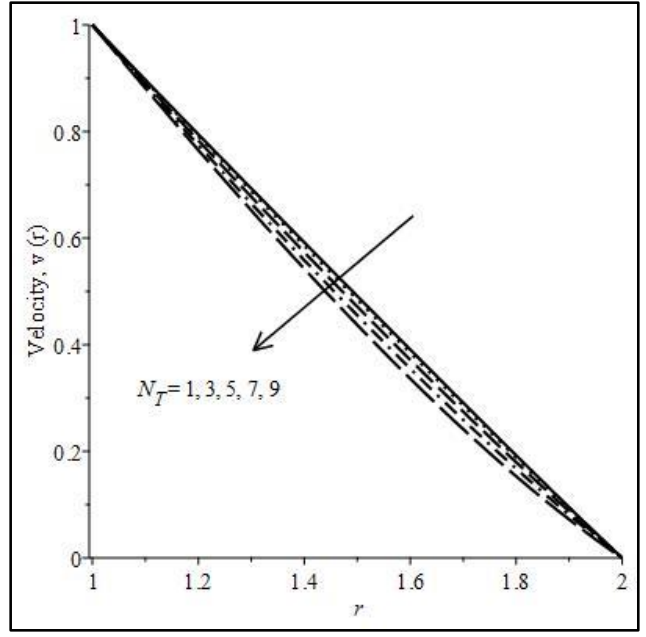

(a)

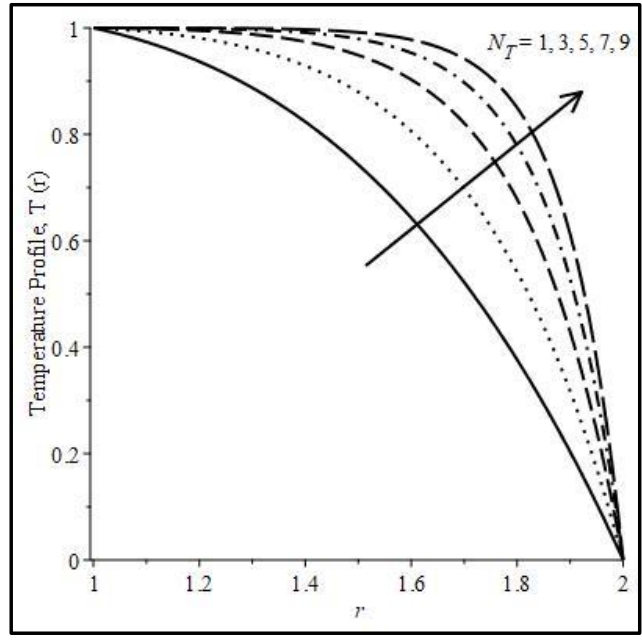

(b)

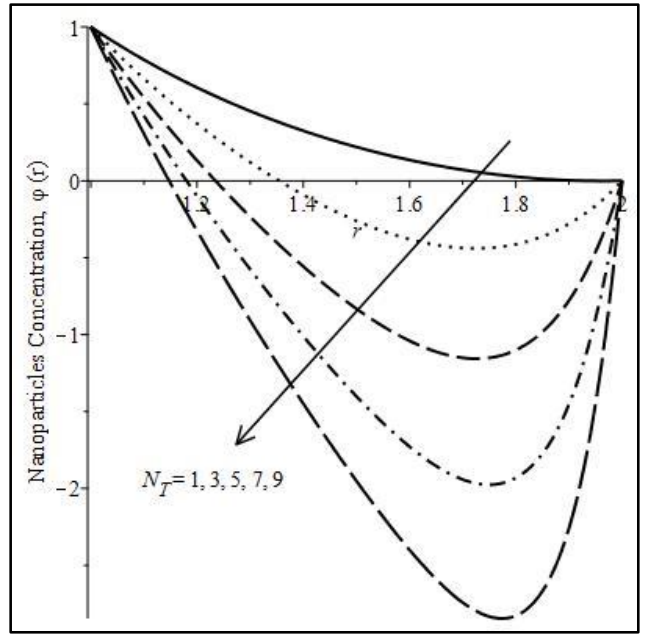

(c) 


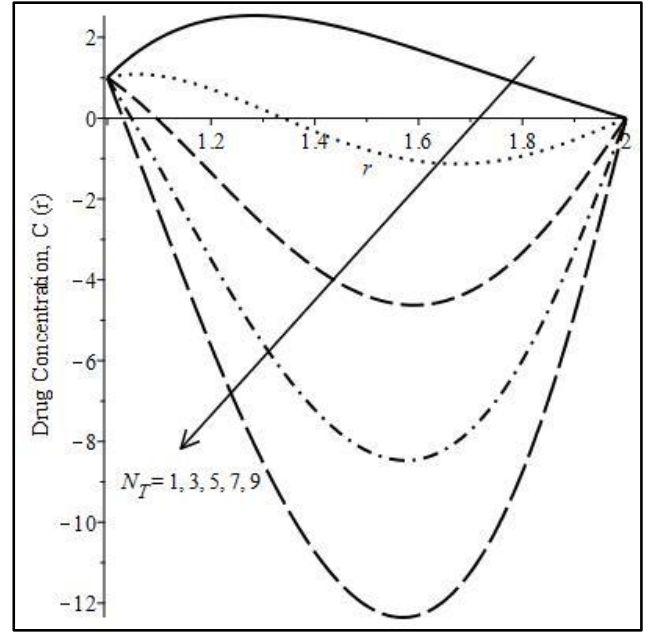

(d)

Figure 3: Effect of thermophoresis parameter, $N_{T}$ on (a) velocity profile, (b) temperature profile, (c) nanoparticles concentration and (d) drug concentration with

$$
\Lambda=B_{r}=G_{r}=\mu_{\mathrm{o}}=M^{2}=P=
$$

$A=B=\alpha=\alpha_{1}=D a_{1}=D a_{2}=1, N_{B}=2$, and $c=-1$.

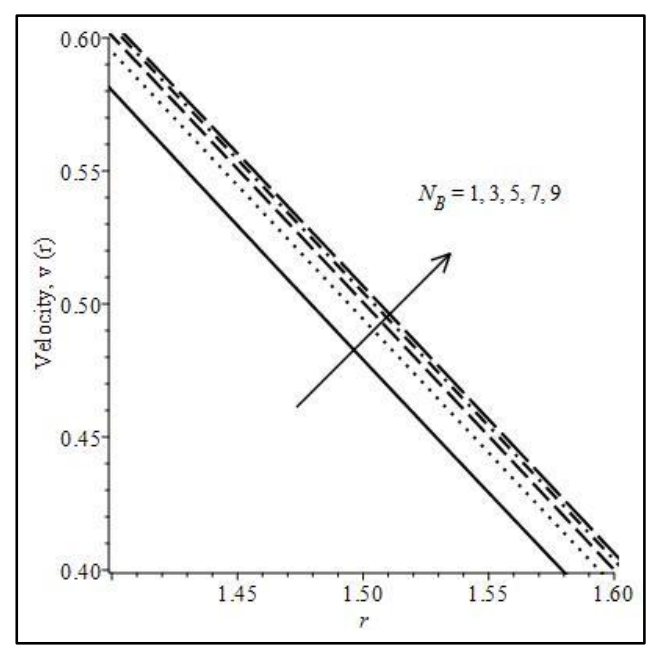

(a)

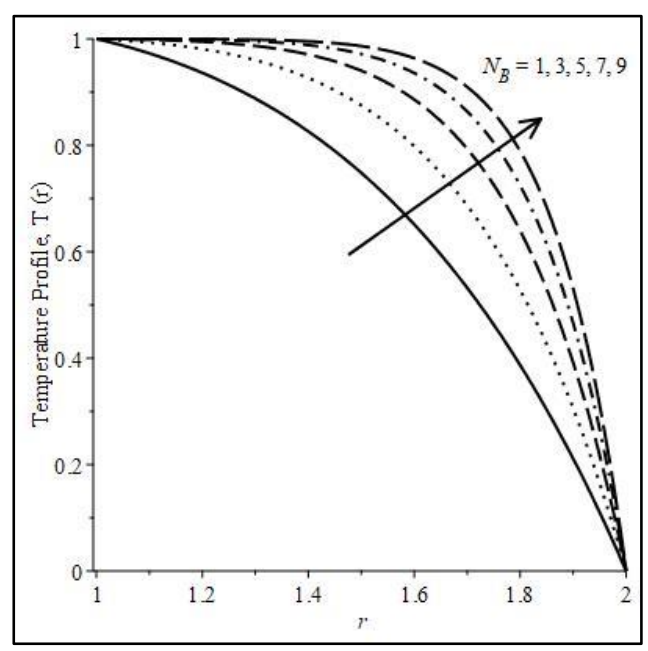

(b)

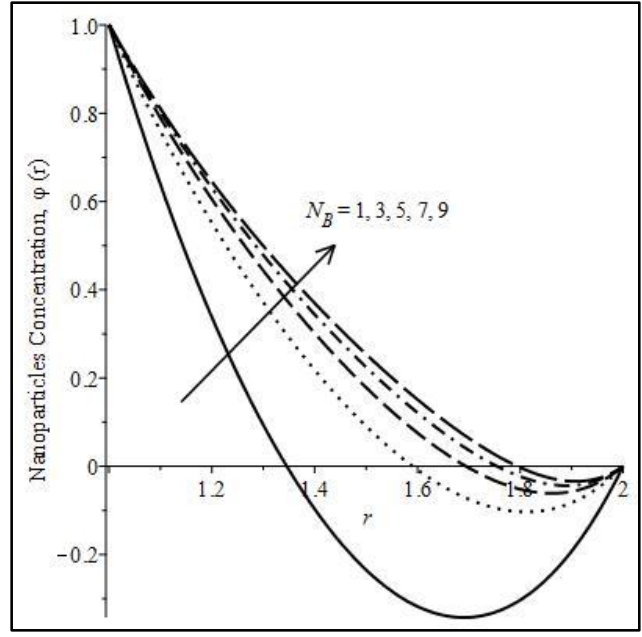

(c)

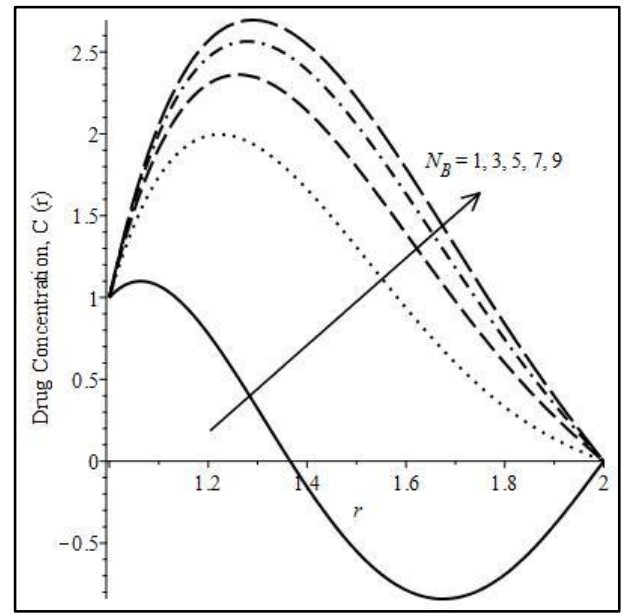

(d)

Figure 4: Effect of Brownian motion parameter, $N_{B}$ on

(a) velocity profile, (b) temperature profile, (c) nanoparticles concentration and (d) drug concentration with $\Lambda=B_{r}=G_{r}=\mu_{\mathrm{O}}=M^{2}=P=$ $A=B=\alpha=\alpha_{1}=D a_{1}=D a_{2}=1, N_{T}=2$, and $c=-1$.

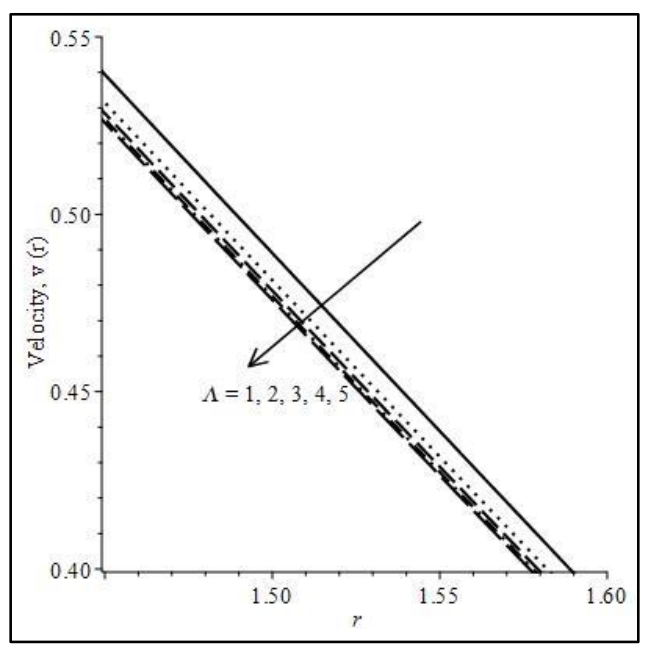

(a) 


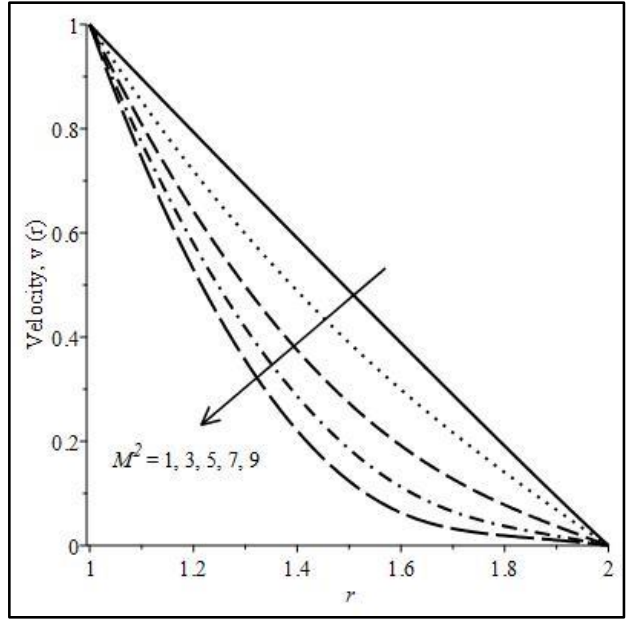

(b)

Figure 5: Effect of (a) third grade parameter, $\Lambda$ and (b)

Magnetic parameter, $M^{2}$ parameter on velocity profile with

$$
\begin{gathered}
B_{r}=G_{r}=\mu_{\mathrm{o}}=P=A=B=\alpha= \\
\alpha_{1}=D a_{1}=D a_{2}=1, N_{T}=2, N_{B}=2 \text { and } c=-1 .
\end{gathered}
$$

\section{CONCLUSION}

The diffusive, advective and reactive processes in thirdgrade non-Newtonian nanofluids in porous coaxial cylinders were studied. The metabolic decay of drug and nanocarriers were considered as first order reaction with the source of drug concentration by nanocarriers. The following effects are observed as increasing $N_{T}$ leads to the increase in temperature while the velocity and nanoparticles and drug concentrations distribution decrease. The increment of the thermophoresis generates larger mass flux due to temperature gradient, which in turn reduce the nanoparticle concentration and drug concentration near the wall. Increasing the thermophoresis parameter and Brownian motion will increase the temperature. Increasing the Brownian motion or decreasing the thermophoresis parameter, the nanoparticles concentration increases.

\section{ACKNOWLEDGEMENT}

The authors would like to acknowledge the support received from the Universiti Teknologi MARA.

\section{REFERENCES}

Akbarzadeh, P 2016, ‘Pulsatile magneto-hydrodynamic blood flows through porous blood vessels using a third grade non-Newtonian fluids model', Computer Methods and Programs in Biomedicine, vol. 126, pp. 3--19.

Buongiorno, J. 2006, 'Convective transport in nanofluids', Journal of Heat Transfer, vol. 128, no. 3, pp. 240-250.

Char, M. I. \&Chiang, K. T. 1994, Stability analysis of BernardMarangoni convection in fluids with internal heat generation.J. Phys. D:Appl., 27, 748-755.

Ellahi, R, Raza, M \& Vafai, K 2012, 'Series solutions of nonNewtonian nanofluids with Reynolds' model and Vogel's model by means of the homotopy analysis method', Mathematical and Computer Modelling, vol. 55, no. 7, pp. 1876-1891.
Ellahi, R, Zeeshan, A, Vafai, K \& Rahman, HU 2011, 'Series solutions for magnetohydrodynamic flow of non-Newtonian nanofluid and heat transfer in coaxial porous cylinder with slip conditions' , Proceedings of the Institution of Mechanical Engineers, Part N:Journal of Nanoengineering and Nanosystems, DOI: 10.1177/1740349911429759, pp. 123-132.

Fosdick, RL \& Rajagopal, KR 1980, 'Thermodynamics and stability of fluids of third grade', Proceedings of the Royal Society of London A: Mathematical, Physical and Engineering Sciences, vol. 369, pp. 351--377.

Hatami, M \& Ganji, DD 2013, 'Heat transfer and flow analysis for $\mathrm{SA}^{-\mathrm{TiO}_{2}}$ non-Newtonian nanofluid passing through the porous media between two coaxial cylinders', Journal of Molecular Liquids, vol.188, pp. 155-161. 
Hatami, M \& Ganji, DD 2014, `Natural convection of sodium alginate (SA) non-Newtonian nanofluid flow between two vertical flat plates by analytical and numerical methods', Case Studies in Thermal Engineering, vol. 2, pp. 14-22.

Hatami, M, Hatami, J \& Ganji, DD 2014, 'Computer simulation of MHD blood conveying gold nanoparticles as a third grade non-Newtonian nanofluid in a hollow porous vessel', Computer Methods and Programs in Biomedicine, vol. 113, no.12, pp. 632-641.

Hayat, T, Shafiq, A \& Alsaedi A 2015, 'MHD axisymmetric flow of third grade fluid by a stretching cylinder', Alexandria Engineering Journal, vol. 54, pp. 205 - 212.

Hayat, T, Waqas, M, Shehzad, SA\& Alsaedi, A 2016, 'Chemically reactive flow of third grade fluid by an exponentially convected stretching sheet', Journal of Molecular Liquids, vol. 223, pp. 853--860.

Hossain, SS , Hossainy, SF , Bazilevs, Y ,Calo, VM \& Hughes, TJ 2012, 'Mathematical modeling of coupled drug and drug-encapsulated nanoparticle transport in patientspecific coronary artery walls', Computational Mechanics, vol. 49, no. 2, pp. 213-242.

Kuznetsov, AV \& Nield, DA 2010, 'Natural convective boundary-layer flow of a nanofluid past a vertical plate', International Journal of Thermal Sciences, vol.49, no.2, pp. 243-247.

Mekheimer, KS, Hasona, WM, Abo-Elkhair, RE \&Zaher AZ 2018, 'Peristaltic blood flow with gold nanoparticles as a third grade nanofluid in catheter: Application of cancer therapy', Physics Letters A, vol. 2, pp. 85 - 93.

Siepmann, J \& G"opferich, A 2001, 'Mathematical modeling of bioerodible, polymeric drug delivery systems', Advanced Drug Delivery Reviews, vol. 48 , pp. 229-247.

Usman, M , Mohyud Din, ST, Zubair, T, Hamid, M \& Wang, W 2018, 'Fluid flow and heat transfer investigation of blood with nanoparticles through porous vessels in the presence of magnetic field', Journal of Algorithms \\& Computational Technology, doi:10.1177/1748301818788661, pp. 1-15. 